\title{
EXISTENCE OF SOLUTIONS \\ FOR A CLASS OF $p(x)$-LAPLACIAN EQUATIONS \\ INVOLVING A CONCAVE-CONVEX NONLINEARITY WITH CRITICAL GROWTH IN $\mathbb{R}^{N}$
}

\author{
Claudianor O. Alves - Marcelo C. Ferreira
}

\begin{abstract}
We prove the existence of solutions for a class of quasilinear problems involving variable exponents and with nonlinearity having critical growth. The main tool used is the variational method, more precisely, Ekeland's Variational Principle and the Mountain Pass Theorem.
\end{abstract}

\section{Introduction}

The present paper concerns with the existence of solutions for the following class of quasilinear problems involving variable exponents

(P) $\begin{cases}-\Delta_{p(x)} u+V(x) u^{p(x)-1}=\lambda h(x) u^{r(x)-1}+\mu u^{q(x)-1}+u^{p^{*}(x)-1}, & \mathbb{R}^{N}, \\ u \geq 0 \text { and } u \neq 0, & \mathbb{R}^{N}, \\ u \in W^{1, p(x)}\left(\mathbb{R}^{N}\right), & \end{cases}$

where $\Delta_{p(x)}$ is the $p(x)$-Laplacian operator given by

$$
\Delta_{p(x)} u=\operatorname{div}\left(|\nabla u|^{p(x)-2} \nabla u\right),
$$

2000 Mathematics Subject Classification. 35A15, 35H30, 35B33.

Key words and phrases. Variational Methods, $p(x)$-Laplacian, critical growth.

Partially supported by INCT-MAT and PROCAD.

C.O. Alves was partially supported by CNPq/Brazil 303080/2009-4. 
$\lambda, \mu$ are positive parameters, $p: \mathbb{R}^{N} \rightarrow \mathbb{R}$ is a Lipschitz continuous function, $V, q, r: \mathbb{R}^{N} \rightarrow \mathbb{R}$ are continuous functions and $h$ is a nonnegative function in $L^{\Theta(x)}\left(\mathbb{R}^{N}\right)$ with

$$
\Theta(x)=\frac{N p(x)}{N p(x)-r(x)(N-p(x))} .
$$

Moreover, the functions $p, q$ and $V$ are $\mathbb{Z}^{N}$-periodic, that is

$$
p(x+y)=p(x), \quad q(x+y)=q(x), \quad V(x+y)=V(x),
$$

for all $x \in \mathbb{R}^{N}$ and for all $y \in \mathbb{Z}^{N}$, and we also assume that

$$
1<p_{-} \leq p(x) \leq p_{+}<N \quad \text { for all } x \in \mathbb{R}^{N} .
$$

$$
1<r_{-} \leq r_{+}<p_{-} \leq p_{+}<q_{-} \leq q(x) \ll p^{*}(x), \quad \text { for all } x \in \mathbb{R}^{N} \text {. }
$$

$$
\inf _{x \in \mathbb{R}^{N}} V(x)=V_{0}>0 .
$$

Here, the notation $u(x) \ll v(x)$ means that $\inf _{x \in \mathbb{R}^{N}}(u(x)-v(x))>0, u_{-}=$ ess $\inf _{x \in \mathbb{R}^{N}} u(x), u_{+}=\operatorname{ess} \sup _{x \in \mathbb{R}^{N}} u(x)$ and $u^{*}(x)=N u(x) /(N-u(x))$ for all $x \in \mathbb{R}^{N}$.

Partial Differential Equations involving the $p(x)$-Laplacian arise, for instance, as a mathematical model for problems involving electrorheological fluids and image restorations, see [1], [2], [10], [14], [15], [37]. This explains the intense research on this subject in the last decades. Regarding to the application of variational methods in order to solve $p(x)$-Laplacian problems, many research were already done when the nonlinearities have a subcritical growth, see for example, [5], [8], [12], [16]-[18], [20], [23], [34] and references therein. However, when the growth involves some criticality, some articles just began appear recently, see the papers due to Alves and Souto [8], Alves [6], Alves andFerreira [7], Bonder and Silva [26], Bonder, Saintier and Silva [24], [25], Fu and Zhang [27], [28], Shang amd Wang [38] and references therein.

In [3], Alves has studied the existence of solutions for the following class of quasilinear problems:

$$
\left\{\begin{array}{l}
-\Delta_{p} u=\lambda g(x) u^{r-1}+u^{p^{*}-1}, \quad \mathbb{R}^{N}, \\
u \geq 0, \quad u \neq 0, \\
u \in D^{1, p}\left(\mathbb{R}^{N}\right),
\end{array}\right.
$$

where $\lambda>0,2 \leq p \leq N, 1<r<p$ and $g$ is a nonnegative function belonging to $L^{\theta}\left(\mathbb{R}^{N}\right)$ with $\theta=N p /(N p-r(N-p))$.

In [3], by using variational methods, more precisely, Mountain Pass Theorem and Ekeland's Variational Principle, the existence of two solutions has been established when $\lambda$ is small enough. In the literature, we can find a lot of papers related to problem $\left(\mathrm{P}_{0}\right)$ involving bounded or unbounded domains, see for example, [9], [11], [13], [30], [31], [36], [39], [40]. However, involving variable 
exponents, the authors know only the paper [26], where the nonlinearity has a behavior like concave-convex and the domain is bounded.

Motivated by the above informations, we prove that similar results to that found in [3] also hold for the case where the exponents are variable. More precisely, we have showing that the energy functional $I: W^{1, p(x)}\left(\mathbb{R}^{N}\right) \rightarrow \mathbb{R}$ associated with $(\mathrm{P})$, which is given by

$$
\begin{aligned}
I(u)=\int_{\mathbb{R}^{N}} \frac{1}{p(x)}\left(|\nabla u|^{p(x)}+V(x)|u|^{p(x)}\right)-\lambda \int_{\mathbb{R}^{N}} \frac{h(x)}{r(x)}\left(u^{+}\right)^{r(x)} \\
-\mu \int_{\mathbb{R}^{N}} \frac{1}{q(x)}\left(u^{+}\right)^{q(x)}-\int_{\mathbb{R}^{N}} \frac{1}{p^{*}(x)}\left(u^{+}\right)^{p^{*}(x)},
\end{aligned}
$$

has two critical points for each $\mu$ large enough and $\lambda$ small enough.

Our main theorem is the following:

THEOREM 1.1. There exists $\mu^{\star}>0$ such that for each $\mu \geq \mu^{\star}$, there is $\lambda_{\mu}=\lambda(\mu)>0$ such that problem $(\mathrm{P})$ has two solutions $\Psi_{1}, \Psi_{2} \in W^{1, p(x)}\left(\mathbb{R}^{N}\right)$ with $I\left(\Psi_{2}\right)<0<I\left(\Psi_{1}\right)$, for all $\lambda \in\left(0, \lambda_{\mu}\right)$.

The Theorem 1.1 is an immediate consequence of Theorems 4.3 and 5.3, which were proved in Sections 4 and 5, respectively. In the proof of the above results, we have used a result found in [7], which shows that if $\left(\mathrm{H}_{0}\right)-\left(\mathrm{H}_{2}\right)$ hold, the problem

$\left(\mathrm{P}_{\mu}\right) \quad\left\{\begin{array}{l}-\Delta_{p(x)} u+V(x)|u|^{p(x)-2} u=\mu|u|^{q(x)-2} u+|u|^{p^{*}(x)-2} u, \quad \mathbb{R}^{N}, \\ u \neq 0 \text { and } u \in W^{1, p(x)}\left(\mathbb{R}^{N}\right),\end{array}\right.$

has a ground state solution, that is, the mountain pass level of the energy functional associated with $\left(\mathrm{P}_{\mu}\right)$ is a critical value.

We recall that the energy functional $I_{\mu}: W^{1, p(x)}\left(\mathbb{R}^{N}\right) \rightarrow \mathbb{R}$ associated to $\left(\mathrm{P}_{\mu}\right)$ is given by

$I_{\mu}(u)=\int_{\mathbb{R}^{N}} \frac{1}{p(x)}\left(|\nabla u|^{p(x)}+V(x)|u|^{p(x)}\right)-\mu \int_{\mathbb{R}^{N}} \frac{1}{q(x)}|u|^{q(x)}-\int_{\mathbb{R}^{N}} \frac{1}{p^{*}(x)}|u|^{p^{*}(x)}$.

Thus, if $c_{\mu}$ denotes the mountain pass level of $I_{\mu}$, we say that $\Psi \in W^{1, p(x)}\left(\mathbb{R}^{N}\right)$ is a ground state solution of $\left(\mathrm{P}_{\mu}\right)$ if

$$
I_{\mu}^{\prime}(\Psi)=0 \quad \text { and } \quad I_{\mu}(\Psi)=c_{\mu} .
$$

In [6], the below limit has been proved

$$
c_{\mu} \rightarrow 0, \quad \text { as } \mu \rightarrow+\infty .
$$

The above limit is a key point in our arguments, because in the present paper, we will denote by $\mu_{0}>0$ a number such that

$$
c_{\mu}<\min \left\{\gamma\left(\frac{1}{K}\right)^{1 / \gamma}, \frac{1}{2 K^{p_{+}}} \nu\right\} \text { for all } \mu \geq \mu_{0},
$$


where

$$
\gamma=\frac{1}{p_{+}}-\frac{1}{p_{-}^{*}}, \quad \nu=\frac{1}{p_{+}}-\frac{1}{q_{-}},
$$

and $K \geq 1$ is fixed satisfying

$$
|u|_{p^{*}(x)} \leq K\|u\|, \quad \text { for all } u \in W^{1, p(x)}\left(\mathbb{R}^{N}\right) .
$$

Furthermore, standard arguments work to prove that the ground state solution $\Psi$ of $\left(\mathrm{P}_{\mu}\right)$ can be chosen nonnegative.

Notation. The following notations will be used in the present work:

- $C$ and $C_{i}$ will denote generic positive constant, which may vary from line to line.

- In all the integrals we omit the symbol $d x$.

- $u^{+}(x)=\max \{u(x), 0\}$ and $u^{-}(x)=\min \{u(x), 0\}$.

\section{Variable exponent Lebesgue and Sobolev spaces}

In this section, we recall some results on variable exponent Lebesgue and Sobolev spaces found in [19], [21] and their references.

Let $z \in L^{\infty}\left(\mathbb{R}^{N}\right)$ with $z_{-} \geq 1$. The variable exponent Lebesgue space $L^{z(x)}\left(\mathbb{R}^{N}\right)$ is defined by

$$
L^{z(x)}\left(\mathbb{R}^{N}\right)=\left\{u: \mathbb{R}^{N} \rightarrow \mathbb{R} \mid u \text { is measurable and } \int_{\mathbb{R}^{N}}|u|^{z(x)}<\infty\right\},
$$

endowed with the norm

$$
|u|_{z(x)}=\inf \left\{\lambda>\left.0\left|\int_{\mathbb{R}^{N}}\right| \frac{u}{\lambda}\right|^{z(x)} \leq 1\right\} .
$$

The variable exponent Sobolev space is defined by

$$
W^{1, z(x)}\left(\mathbb{R}^{N}\right)=\left\{u \in L^{z(x)}\left(\mathbb{R}^{N}\right)|| \nabla u \mid \in L^{z(x)}\left(\mathbb{R}^{N}\right)\right\},
$$

with the norm

$$
\|u\|_{1, z(x)}=|u|_{z(x)}+|\nabla u|_{z(x)} .
$$

If $M \in L^{\infty}\left(\mathbb{R}^{N}\right)$ satisfies $M_{-}>0$, the norm

$$
\|u\|=\inf \left\{\lambda>0 \mid \int_{\mathbb{R}^{N}}\left(\left|\frac{\nabla u}{\lambda}\right|^{z(x)}+M(x)\left|\frac{u}{\lambda}\right|^{z(x)}\right) \leq 1\right\}
$$

is equivalent to norm $\|\cdot\|_{1, z(x)}$.

If $z_{-}>1$, the spaces $L^{z(x)}\left(\mathbb{R}^{N}\right)$ and $W^{1, z(x)}\left(\mathbb{R}^{N}\right)$ are reflexive and separable Banach spaces with these norms.

Proposition 2.1. The functional $\xi: W^{1, z(x)}\left(\mathbb{R}^{N}\right) \rightarrow \mathbb{R}$ defined by

$$
\xi(u)=\int_{\mathbb{R}^{N}}\left(|\nabla u|^{z(x)}+M(x)|u|^{z(x)}\right),
$$

has the following properties: 
(a) If $\|u\| \geq 1$, then $\|u\|^{z_{-}} \leq \xi(u) \leq\|u\|^{z_{+}}$.

(b) If $\|u\| \leq 1$, then $\|u\|^{z_{+}} \leq \xi(u) \leq\|u\|^{z_{-}}$.

In particular, for $\left(u_{n}\right) \subset W^{1, z(x)}\left(\mathbb{R}^{N}\right)$,

$$
\left\|u_{n}\right\| \rightarrow 0 \Leftrightarrow \xi\left(u_{n}\right) \rightarrow 0
$$

and

$$
\left(u_{n}\right) \text { is bounded in } W^{1, z(x)}\left(\mathbb{R}^{N}\right) \Leftrightarrow \xi\left(u_{n}\right) \text { is bounded in } \mathbb{R} \text {. }
$$

REMARK 2.2. For the functional $\xi_{z}: L^{z(x)}\left(\mathbb{R}^{N}\right) \rightarrow \mathbb{R}$ given by

$$
\xi_{z}(u)=\int_{\mathbb{R}^{N}}|u|^{z(x)}
$$

the same conclusion of Proposition 2.1 also holds. Moreover, from (a) and (b),

$$
|u|_{z(x)} \leq \max \left\{\left(\int_{\mathbb{R}^{N}}|u|^{z(x)}\right)^{1 / z_{-}},\left(\int_{\mathbb{R}^{N}}|u|^{z(x)}\right)^{1 / z_{+}}\right\} .
$$

Related to the Lebesgue space $L^{z(x)}\left(\mathbb{R}^{N}\right)$, we have the following generalized Hölder's inequality.

Proposition $2.3([35$, p. 9$])$. For $z \in L^{\infty}\left(\mathbb{R}^{N}\right)$ with $z_{-}>1$, let $z^{\prime}: \mathbb{R}^{N} \rightarrow \mathbb{R}$ be such that

$$
\frac{1}{z(x)}+\frac{1}{z^{\prime}(x)}=1 \quad \text { a.e. in } \mathbb{R}^{N} \text {. }
$$

Then, for any $u \in L^{z(x)}\left(\mathbb{R}^{N}\right)$ and $v \in L^{z^{\prime}(x)}\left(\mathbb{R}^{N}\right)$,

$$
\left|\int_{\mathbb{R}^{N}} u v\right| \leq\left(\frac{1}{z_{-}}+\frac{1}{z_{-}^{\prime}}\right)|u|_{z(x)}|v|_{z^{\prime}(x)} .
$$

Proposition 2.4 ([19, Theorems 1.1, 1.3]). Let $z: \mathbb{R}^{N} \rightarrow \mathbb{R}$ be a Lipschitz continuous satisfying $1<z_{-} \leq z_{+}<N$ and $s: \mathbb{R}^{N} \rightarrow \mathbb{R}$ be a measurable function.

(a) If $z \leq s \leq z^{*}$, the embedding $W^{1, z(x)}\left(\mathbb{R}^{N}\right) \hookrightarrow L^{s(x)}\left(\mathbb{R}^{N}\right)$ is continuous.

(b) If $z \leq s \ll z^{*}$, the embedding $W^{1, z(x)}\left(\mathbb{R}^{N}\right) \hookrightarrow L_{\text {loc }}^{s(x)}\left(\mathbb{R}^{N}\right)$ is compact.

The next two results are very important in our arguments and their proofs follows the same arguments explored in [32], this form, we will omit their proofs.

Proposition 2.5 (Brezis-Lieb's lemma, first version). Let $z \in L^{\infty}\left(\mathbb{R}^{N}\right)$ with $z_{-} \geq 1$ and $\left(\eta_{n}\right) \subset L^{z(x)}\left(\mathbb{R}^{N}, \mathbb{R}^{k}\right)$ verifying

(a) $\eta_{n}(x) \rightarrow \eta(x)$, almost everywhere in $\mathbb{R}^{N}$;

(b) $\sup _{n \in \mathbb{N}}\left|\eta_{n}\right|_{L^{z(x)}\left(\mathbb{R}^{N}, \mathbb{R}^{k}\right)}<\infty$.

Then, $\eta \in L^{z(x)}\left(\mathbb{R}^{N}, \mathbb{R}^{k}\right)$ and

$$
\int_{\mathbb{R}^{N}}\left(\left|\eta_{n}\right|^{z(x)}-\left|\eta_{n}-\eta\right|^{z(x)}-|\eta|^{z(x)}\right) d x=o_{n}(1) .
$$


Proposition 2.6 (Brezis-Lieb's lemma, second version). Let $z \in L^{\infty}\left(\mathbb{R}^{N}\right)$ with $z_{-}>1$ and $\left(\eta_{n}\right) \subset L^{z(x)}\left(\mathbb{R}^{N}, \mathbb{R}^{k}\right)$ verifying

(a) $\eta_{n}(x) \rightarrow \eta(x)$, almost everywhere in $\mathbb{R}^{N}$;

(b) $\sup _{n \in \mathbb{N}}\left|\eta_{n}\right|_{L^{z(x)}\left(\mathbb{R}^{N}, \mathbb{R}^{k}\right)}<\infty$.

Then $\eta_{n} \rightarrow \eta$ in $L^{z(x)}\left(\mathbb{R}^{N}, \mathbb{R}^{k}\right)$.

The next proposition is a Brezis-Lieb type result and it applies an important role in our paper. For the case where $z$ is constant, the result is due to Alves [4] for $z \geq 2$ and Mercuri and Willem [33] for $1<z<2$.

Proposition 2.7 (Brezis-Lieb lemma, third version). Let $z \in L^{\infty}\left(\mathbb{R}^{N}\right)$ with $z_{-}>1$ and $\left(\eta_{n}\right)$ a sequence in $L^{z(x)}\left(\mathbb{R}^{N}, \mathbb{R}^{k}\right)$ such that

(a) $\eta_{n}(x) \rightarrow \eta(x)$ almost everywhere in $\mathbb{R}^{N}$;

(b) $\sup _{n \in \mathbb{N}}\left|\eta_{n}\right|_{L^{z(x)}\left(\mathbb{R}^{N}, \mathbb{R}^{k}\right)}<\infty$.

Then

$$
\left.\int_{\mathbb{R}^{N}}|| \eta_{n}\right|^{z(x)-2} \eta_{n}-\left|\eta_{n}-\eta\right|^{z(x)-2}\left(\eta_{n}-\eta\right)-\left.|\eta|^{z(x)-2} \eta\right|^{z^{\prime}(x)}=o_{n}(1) .
$$

Proof. In what follows, we set $A(x, y)=|y|^{z(x)-2} y$, for all $x \in \mathbb{R}^{N}, y \in \mathbb{R}^{k}$. Our goal is to show that

$$
\begin{array}{r}
\int_{\left\{x \in \mathbb{R}^{N} ; 1<z(x)<2\right\}}\left|A\left(x, \eta_{n}(x)\right)-A\left(x, \eta_{n}(x)-\eta(x)\right)-A(x, \eta(x))\right|^{z^{\prime}(x)} \\
=o_{n}(1)
\end{array}
$$

and

$$
\begin{array}{r}
\int_{\left\{x \in \mathbb{R}^{N} ; z(x) \geq 2\right\}}\left|A\left(x, \eta_{n}(x)\right)-A\left(x, \eta_{n}(x)-\eta(x)\right)-A(x, \eta(x))\right|^{z^{\prime}(x)} \\
=o_{n}(1),
\end{array}
$$

because if the above limits occur, we have that (2.5) also occurs. This way, we will begin showing the limit $(2.6)$. If the set $z^{-1}((1,2))$ has zero measure, we have nothing to do. Thereby, we will assume that $z^{-1}((1,2))$ has a positive measure and we will adapt the ideas found in [33]. First of all, we observe that

$$
\alpha=\sup _{\substack{x \in z^{-1}((1,2)) \\ y, h \in \mathbb{R}^{k} \\ h \neq 0}} F(x, y, h)<\infty,
$$

where

$$
F(x, y, h)=\left|\frac{|y+h|^{z(x)-2}(y+h)-|y|^{z(x)-2} y}{|h|^{z(x)-1}}\right| .
$$

In fact, given any $t>0$, it is easy to see that

$$
F(x, y, t h)=F\left(x, \frac{y}{t}, h\right),
$$


hence

$$
\alpha=\sup _{\substack{x \in z^{-1}((1,2)) \\ y, h \in \mathbb{R}^{k} \\|h|=1}} F(x, y, h) .
$$

Firstly, if $|y| \leq 2$, for any $x \in z^{-1}((1,2)), h \in \mathbb{R}^{k}$ with $|h|=1$, it follows that

$$
|| y+\left.h\right|^{z(x)-2}(y+h)-|y|^{z(x)-2} y \mid \leq 5,
$$

implying that

$$
\alpha_{1}=\sup _{\substack{x \in z^{-1}((1,2)) \\ y, h \in \mathbb{R}^{k} \\|y| \leq 2,|h|=1}} F(x, y, h)<\infty .
$$

On the other hand, if $|y|>2$, for any $t \in[0,1]$ and $h \in \mathbb{R}^{k}$ with $|h|=1$, it holds

$$
|y+t h| \geq|y|-t|h|>1 \text {. }
$$

Therefore, for each $i=1, \ldots, k$ and $x \in z^{-1}((1,2))$,

$$
\begin{aligned}
& || y+\left.h\right|^{z(x)-2}\left(y_{i}+h_{i}\right)-|y|^{z(x)-2} y_{i}|=| \int_{0}^{1} \frac{d}{d t}|y+t h|^{z(x)-2}\left(y_{i}+t h_{i}\right) d t \mid \\
& =\left|\int_{0}^{1}\left(|y+t h|^{z(x)-2} h_{i}+(z(x)-2)\left(y_{i}+t h_{i}\right)|y+t h|^{z(x)-4}(y+t h) \cdot h\right) d t\right| \\
& \leq(3-z(x)) \int_{0}^{1}|y+t h|^{z(x)-2} d t<2 \int_{0}^{1} 1 d t=2
\end{aligned}
$$

showing that

$$
\alpha_{2}=\sup _{\substack{x \in z^{-1}((1,2)) \\ y, h \in \mathbb{R}^{k} \\|y|>2,|h|=1}} F(x, y, h)<\infty .
$$

Combining (2.9) with (2.10), we obtain (2.8).

A direct computation gives

$$
\begin{aligned}
& \left|A\left(x, \eta_{n}(x)\right)-A\left(x, \eta_{n}(x)-\eta(x)\right)-A(x, \eta(x))\right| \\
& \leq F\left(x, \eta_{n}(x)-\eta(x), \eta(x)\right)|\eta(x)|^{z(x)-1}+|\eta(x)|^{z(x)-1} \leq(\alpha+1)|\eta(x)|^{z(x)-1},
\end{aligned}
$$

for all $x \in z^{-1}((1,2))$, and so,

$$
\left|A\left(x, \eta_{n}(x)\right)-A\left(x, \eta_{n}(x)-\eta(x)\right)-A(x, \eta(x))\right|^{z^{\prime}(x)} \leq(\alpha+1)^{z_{+}^{\prime}}|\eta(x)|^{z(x)},
$$

for all $x \in z^{-1}((1,2))$, where $z^{\prime}(x)=z(x) /(z(x)-1)$, for all $x \in \mathbb{R}^{N}$. Now, the limit (2.6) follows from the last inequality together with Lebesgue's dominated convergence theorem.

In the proof of (2.7), we will adapt the ideas found in [4]. If the set $z^{-1}([2, \infty))$ has zero measure, we have nothing to do. Thereby, we will assume 
that $z^{-1}([2, \infty))$ has a positive measure. For each $i=1, \ldots, k$ and $x \in \mathbb{R}^{N}$, we have that

$$
\begin{aligned}
A_{i}\left(x, \eta_{n}(x)\right)- & A_{i}\left(x, \eta_{n}(x)-\eta(x)\right) \\
& =\left|\eta_{n}(x)\right|^{z(x)-2} \eta_{n}^{i}(x)-\left|\eta_{n}(x)-\eta(x)\right|^{z(x)-2}\left(\eta_{n}^{i}(x)-\eta_{i}(x)\right) .
\end{aligned}
$$

So, by the previous calculations,

$$
\begin{aligned}
\mid A_{i}\left(x, \eta_{n}(x)\right) & -A_{i}\left(x, \eta_{n}(x)-\eta(x)\right) \mid \\
\leq & (z(x)-1)|\eta(x)| \int_{0}^{1}\left|\eta_{n}(x)+(t-1) \eta(x)\right| z(x)-2 d t \\
\leq & \left(z_{+}-1\right)|\eta(x)|\left(\left|\eta_{n}(x)\right|+|\eta(x)|\right)^{z(x)-2} .
\end{aligned}
$$

Therefore

$$
\left|A\left(x, \eta_{n}(x)\right)-A\left(x, \eta_{n}(x)-\eta(x)\right)\right| \leq C\left(|\eta(x)|^{z(x)-1}+|\eta(x)|\left|\eta_{n}(x)\right|^{z(x)-2}\right),
$$

for all $x \in z^{-1}([2, \infty))$. The above inequality combined with Young's inequality leads, for all $\varepsilon>0$, to

$$
\left|A\left(x, \eta_{n}(x)\right)-A\left(x, \eta_{n}(x)-\eta(x)\right)\right| \leq C(\varepsilon)|\eta(x)|^{z(x)-1}+\varepsilon\left|\eta_{n}(x)\right|^{z(x)-1} .
$$

Now, for each $\varepsilon>0, n \in \mathbb{N}$, we define the function $f_{\varepsilon, n}: \mathbb{R}^{N} \rightarrow \mathbb{R}$ given by $f_{\varepsilon, n}(x)=\max \left\{\left|A\left(x, \eta_{n}(x)\right)-A\left(x, \eta_{n}(x)-\eta(x)\right)-A(x, \eta(x))\right|-\varepsilon\left|\eta_{n}(x)\right|^{z(x)-1}, 0\right\}$, which satisfies $f_{\varepsilon, n}(x) \rightarrow 0$ almost everywhere in $z^{-1}([2, \infty))$, as $n \rightarrow \infty$, and

$$
0 \leq f_{\varepsilon, n}(x) \leq(C(\varepsilon)+1)|\eta(x)|^{z(x)-1}, \quad \text { for all } x \in z^{-1}([2, \infty)) .
$$

So, by Lebesgue's dominated convergence theorem,

$$
\int_{z^{-1}([2, \infty))} f_{\varepsilon, n}^{z^{\prime}(x)} \rightarrow 0, \quad \text { as } n \rightarrow \infty .
$$

On the other hand, by the definition of $f_{\varepsilon, n}$,

$$
\left|A\left(x, \eta_{n}(x)\right)-A\left(x, \eta_{n}(x)-\eta(x)\right)-A(x, \eta(x))\right| \leq \varepsilon\left|\eta_{n}(x)\right|^{z(x)-1}+f_{\varepsilon, n}(x),
$$

for all $x \in \mathbb{R}^{N}$. Consequently,

$$
\begin{aligned}
\mid A\left(x, \eta_{n}(x)\right)-A\left(x, \eta_{n}(x)-\eta(x)\right)-A(x, \eta(x)) & \left.\right|^{z^{\prime}(x)} \\
& \leq 2^{z_{+}^{\prime}\left(\varepsilon^{z_{-}^{\prime}}\left|\eta_{n}(x)\right|^{z(x)}+f_{\varepsilon, n}^{z^{\prime}(x)}\right),}
\end{aligned}
$$

for all $x \in \mathbb{R}^{N}$ and $\varepsilon>0$ sufficiently small. Thus,

$$
\begin{aligned}
\varlimsup_{n} \int_{z^{-1}([2, \infty))} \mid A\left(x, \eta_{n}(x)\right)-A(x, & \left.\eta_{n}(x)-\eta(x)\right)-\left.A(x, \eta(x))\right|^{z^{\prime}(x)} \\
& \leq 2^{z_{+}^{\prime} \varepsilon^{z_{-}^{\prime}}} \int_{z^{-1}([2, \infty))}\left|\eta_{n}(x)\right|^{z(x)} \leq C \varepsilon^{z_{-}^{\prime}},
\end{aligned}
$$

for all $\varepsilon>0$, which implies that (2.7) holds. 


\section{Preliminary results}

In what follows, we will consider on $W^{1, p(x)}\left(\mathbb{R}^{N}\right)$ the following norm

$$
\|u\|=\inf \left\{\alpha>0 ; \rho\left(\alpha^{-1} u\right) \leq 1\right\}
$$

with

$$
\rho(u)=\int_{\mathbb{R}^{N}}\left(|\nabla u|^{p(x)}+V(x)|u|^{p(x)}\right) .
$$

Using well known arguments, we have that the energy functional

$$
I: W^{1, p(x)}\left(\mathbb{R}^{N}\right) \rightarrow \mathbb{R}
$$

associated with $(\mathrm{P})$, which is given by

$$
\begin{aligned}
I(u)= & \int_{\mathbb{R}^{N}} \frac{1}{p(x)}\left(|\nabla u|^{p(x)}+V(x)|u|^{p(x)}\right) \\
& -\lambda \int_{\mathbb{R}^{N}} \frac{h(x)}{r(x)}\left(u^{+}\right)^{r(x)}-\mu \int_{\mathbb{R}^{N}} \frac{1}{q(x)}\left(u^{+}\right)^{q(x)}-\int_{\mathbb{R}^{N}} \frac{1}{p^{*}(x)}\left(u^{+}\right)^{p^{*}(x)},
\end{aligned}
$$

is well defined and $I \in C^{1}\left(W^{1, p(x)}\left(\mathbb{R}^{N}\right), \mathbb{R}\right)$ with

$$
\begin{aligned}
I^{\prime}(u) v= & \int_{\mathbb{R}^{N}}\left(|\nabla u|^{p(x)-2} \nabla u \nabla v+V(x)|u|^{p(x)-2} u v\right) \\
& -\lambda \int_{\mathbb{R}^{N}} h(x)\left(u^{+}\right)^{r(x)-1} v-\mu \int_{\mathbb{R}^{N}}\left(u^{+}\right)^{q(x)-1} v-\int_{\mathbb{R}^{N}}\left(u^{+}\right)^{p^{*}(x)-1} v,
\end{aligned}
$$

for all $u, v \in W^{1, p(x)}\left(\mathbb{R}^{N}\right)$.

Lemma 3.1. All $(\mathrm{PS})_{d}$ sequences $\left(v_{n}\right)$ for I are bounded. Furthermore, $\left(v_{n}^{+}\right)$ is a $(\mathrm{PS})_{d}$ sequence for $I$.

Proof. If there exist only a finite number of terms $\left(v_{n}\right)$ such that $\rho\left(v_{n}\right)>1$, then $\left(v_{n}\right)$ is bounded and the proof is complete. Otherwise, suppose the existence of a infinitely many terms of $\left(v_{n}\right)$ such that $\rho\left(v_{n}\right)>1$. Since $\left(v_{n}\right)$ is a $(\mathrm{PS})_{d}$ sequence, there is $n_{0} \in \mathbb{N}$ such that

$$
I\left(v_{n}\right)-\frac{1}{q_{-}} I^{\prime}\left(v_{n}\right) v_{n} \leq d+1+\left\|v_{n}\right\|, \quad n \geq n_{0} .
$$

On the other hand, using the fact that $\rho\left(v_{n}\right)>1$ and Hölder's inequality, we get

$$
\begin{aligned}
& I\left(v_{n}\right)-\frac{1}{q_{-}} I^{\prime}\left(v_{n}\right) v_{n} \\
& \quad \geq\left(\frac{1}{p_{+}}-\frac{1}{q_{-}}\right)\left\|v_{n}\right\|^{p_{-}}-\lambda\left(\frac{1}{r_{-}}-\frac{1}{q_{-}}\right) \int_{\mathbb{R}^{N}} h(x)\left|v_{n}\right|^{r(x)} \\
& \quad \geq\left(\frac{1}{p_{+}}-\frac{1}{q_{-}}\right)\left\|v_{n}\right\|^{p_{-}}-\left.\left.\lambda\left(\frac{1}{r_{-}}-\frac{1}{q_{-}}\right) C|h|_{\Theta(x)}|| v_{n}\right|^{r(x)}\right|_{p^{*}(x) / r(x)},
\end{aligned}
$$


and so,

$$
\begin{aligned}
I\left(v_{n}\right) & -\frac{1}{q_{-}} I^{\prime}\left(v_{n}\right) v_{n} \\
& \geq\left(\frac{1}{p_{+}}-\frac{1}{q_{-}}\right)\left\|v_{n}\right\|^{p_{-}}-\lambda\left(\frac{1}{r_{-}}-\frac{1}{q_{-}}\right) C|h|_{\Theta(x)}\left(\left|v_{n}\right|_{p^{*}(x)}^{r_{-}}+\left|v_{n}\right|_{p^{*}(x)}^{r_{+}}\right) \\
& \geq\left(\frac{1}{p_{+}}-\frac{1}{q_{-}}\right)\left\|v_{n}\right\|^{p_{-}}-\lambda\left(\frac{1}{r_{-}}-\frac{1}{q_{-}}\right)|h|_{\Theta(x)}\left(C_{1}\left\|v_{n}\right\|^{r_{-}}+C_{2}\left\|v_{n}\right\|^{r_{+}}\right) .
\end{aligned}
$$

From this, for $n \geq n_{0}$,

$$
\begin{aligned}
d+1+ & \left\|v_{n}\right\| \\
& \geq\left(\frac{1}{p_{+}}-\frac{1}{q_{-}}\right)\left\|v_{n}\right\|^{p_{-}}-\lambda\left(\frac{1}{r_{-}}-\frac{1}{q_{-}}\right)|h|_{\Theta(x)}\left(C_{1}\left\|v_{n}\right\|^{r_{-}}+C_{2}\left\|v_{n}\right\|^{r_{+}}\right)
\end{aligned}
$$

which yields $\left(v_{n}\right)$ is also bounded in this case.

Now, we will prove that $\left(v_{n}^{+}\right)$is also a (PS $)_{d}$ sequence for $I$. Note that the boundedness of $\left(v_{n}^{-}\right)$combined with the limit $\left\|I^{\prime}\left(v_{n}\right)\right\| \rightarrow 0$ gives $I^{\prime}\left(v_{n}\right) v_{n}^{-} \rightarrow 0$, from where it follows that $\rho\left(v_{n}^{-}\right) \rightarrow 0$, or equivalently $v_{n}^{-} \rightarrow 0$ in $W^{1, p(x)}\left(\mathbb{R}^{N}\right)$. Now, a simple computation yields

$$
I\left(v_{n}\right)=I\left(v_{n}^{+}\right)+o_{n}(1) \quad \text { and } \quad I^{\prime}\left(v_{n}\right)=I^{\prime}\left(v_{n}^{+}\right)+o_{n}(1),
$$

proving that $\left(v_{n}^{+}\right)$is a $(\mathrm{PS})_{d}$ sequence.

From the last lemma, hereafter we will assume that all $(\mathrm{PS})_{d}$ sequences for $I$ are composed by nonnegative functions. Moreover, once that $W^{1, p(x)}\left(\mathbb{R}^{N}\right)$ is reflexive, if $\left(v_{n}\right)$ is a $(\mathrm{PS})_{d}$ sequence for $I$, we also assume that for some subsequence, still denoted by itself, there is $v \in W^{1, p(x)}\left(\mathbb{R}^{N}\right)$ such that

$$
v_{n} \rightarrow v \quad \text { in } W^{1, p(x)}\left(\mathbb{R}^{N}\right), \quad v_{n}(x) \rightarrow v(x) \quad \text { a.e in } \mathbb{R}^{N}, \quad v(x) \geq 0 \quad \text { a.e in } \mathbb{R}^{N} .
$$

The next lemma is a key point in our arguments, which can be found in [7]. However for the reader's convenience we will make its proof.

Lemma 3.2. Let $\left(v_{n}\right)$ be a $(\mathrm{PS})_{d}$ sequence for $I$ and $v \in W^{1, p(x)}\left(\mathbb{R}^{N}\right)$ such that $v_{n} \rightarrow v$ in $W^{1, p(x)}\left(\mathbb{R}^{N}\right)$. Then, $I^{\prime}(v)=0$. Hence, if $v \neq 0$, $v$ is a nontrivial solution for $(\mathrm{P})$.

Proof. Following a standard reasoning, it is sufficient to show that, up to a subsequence,

$$
\nabla v_{n}(x) \rightarrow \nabla v(x) \text { a.e in } \mathbb{R}^{N} .
$$

We begin observing that, up to a subsequence, there exist two nonnegative measures $\mathfrak{m}$ and $\mathfrak{n}$ in $\mathcal{M}\left(\mathbb{R}^{N}\right)$ such that

$$
\begin{array}{cc}
\left|\nabla v_{n}\right|^{p(x)} \rightarrow \mathfrak{m} & \text { in } \mathcal{M}\left(\mathbb{R}^{N}\right), \\
\left|v_{n}\right|^{p^{*}(x)} \rightarrow \mathfrak{n} & \text { in } \mathcal{M}\left(\mathbb{R}^{N}\right) .
\end{array}
$$


In this case, according a concentration compactness principle in [27], there exists a countable index set $\mathfrak{I}$ such that

$$
\mathfrak{n}=|v|^{p^{*}(x)} d x+\sum_{i \in \mathfrak{I}} \mathfrak{n}_{i} \delta_{x_{i}}, \quad \mathfrak{m} \geq|\nabla v|^{p(x)} d x+\sum_{i \in \mathfrak{I}} \mathfrak{m}_{i} \delta_{x_{i}},
$$

and

$$
\mathfrak{n}_{i} \leq S \max \left\{\mathfrak{m}_{i}^{p_{+}^{*} / p_{-}}, \mathfrak{m}_{i}^{p_{-}^{*} / p_{+}}\right\}
$$

where $\left(\mathfrak{n}_{i}\right)_{i \in \mathfrak{I}},\left(\mathfrak{m}_{i}\right)_{i \in \mathfrak{I}} \subset[0, \infty)$ and $\left(x_{i}\right)_{i \in \mathfrak{I}} \subset \mathbb{R}^{N}$. The constant $S$ is given by

$$
S=\sup _{\substack{u \in W^{1, p(x)} \\\|u\| \leq 1}} \int_{\left.\mathbb{R}^{N}\right)}|u|^{p^{*}(x)} .
$$

Our first task is to prove that $\mathfrak{m}_{\mathfrak{i}}=\mathfrak{n}_{\mathfrak{i}}$, for all $i \in \mathfrak{I}$. For this, let $\varphi \in C_{0}^{\infty}\left(\mathbb{R}^{N}\right)$ such that $\varphi(x)=1$ in $B_{1}(0), \varphi(x)=0$ in $B_{2}^{c}(0)$ and $0 \leq \varphi(x) \leq 1$, for all $x \in \mathbb{R}^{N}$. Fixed $i \in \mathfrak{I}$, we consider, for each $\varepsilon>0$,

$$
\varphi_{\varepsilon}(x)=\varphi\left(\frac{x-x_{i}}{\varepsilon}\right) \quad \text { for all } x \in \mathbb{R}^{N} .
$$

Since $\left(v_{n}\right)$ is bounded in $W^{1, p(x)}\left(\mathbb{R}^{N}\right)$, the sequence $\left(\varphi_{\varepsilon} v_{n}\right)$ is also bounded in $W^{1, p(x)}\left(\mathbb{R}^{N}\right)$. Thus, $I^{\prime}\left(v_{n}\right)\left(\varphi_{\varepsilon} v_{n}\right)=o_{n}(1)$, that is,

$$
\begin{aligned}
\int_{\mathbb{R}^{N}}\left(\varphi_{\varepsilon}\left|\nabla v_{n}\right|^{p(x)}+v_{n}\left|\nabla v_{n}\right|^{p(x)-2} \nabla v_{n} \nabla \varphi_{\varepsilon}\right)+\int_{\mathbb{R}^{N}} V(x)\left|v_{n}\right|^{p(x)} \varphi_{\varepsilon} \\
\quad=\lambda \int_{\mathbb{R}^{N}} h(x)\left|v_{n}\right|^{r(x)} \varphi_{\varepsilon}+\mu \int_{\mathbb{R}^{N}}\left|v_{n}\right|^{q(x)} \varphi_{\varepsilon}+\int_{\mathbb{R}^{N}}\left|v_{n}\right|^{p^{*}(x)} \varphi_{\varepsilon}+o_{n}(1) .
\end{aligned}
$$

Taking the limits as $n \rightarrow \infty$, the weak convergence of $\left(\left|\nabla v_{n}\right|^{p(x)}\right)$ and $\left(\left|v_{n}\right|^{p^{*}(x)}\right)$ in $\mathcal{M}\left(\mathbb{R}^{N}\right)$ combined with the Lebesgue's dominated convergence theorem and Proposition 2.6, give us

$$
\begin{array}{r}
\int_{\mathbb{R}^{N}} \varphi_{\varepsilon} d \mathfrak{m}+\limsup _{n} \int_{\mathbb{R}^{N}} v_{n}\left|\nabla v_{n}\right|^{p(x)-2} \nabla v_{n} \nabla \varphi_{\varepsilon}+\int_{\mathbb{R}^{N}} V(x)|v|^{p(x)} \varphi_{\varepsilon} \\
=\lambda \int_{\mathbb{R}^{N}} h(x)|v|^{r(x)} \varphi_{\varepsilon}+\mu \int_{\mathbb{R}^{N}}|v|^{q(x)} \varphi_{\varepsilon}+\int_{\mathbb{R}^{N}} \varphi_{\varepsilon} d \mathfrak{n} .
\end{array}
$$

Using Hölder's inequality and the boundedness of $\left(v_{n}\right)$ in $W^{1, p(x)}\left(\mathbb{R}^{N}\right)$,

$$
\begin{aligned}
& \left.\left|\int_{\mathbb{R}^{N}} v_{n}\right| \nabla v_{n}\right|^{p(x)-2} \nabla v_{n} \cdot \nabla \varphi_{\varepsilon} \mid \\
& \leq \int_{\mathbb{R}^{N}}\left|\nabla v_{n}\right|^{p(x)-1}\left|v_{n} \nabla \varphi_{\varepsilon}\right| \leq\left.\left. C|| \nabla v_{n}\right|^{p(x)-1}\right|_{p^{\prime}(x)}\left|v_{n}\right| \nabla \varphi_{\varepsilon} \|_{p(x)} \\
& \leq C \max \left\{\left(\int_{\mathbb{R}^{N}}\left|v_{n}\right|^{p(x)}\left|\nabla \varphi_{\varepsilon}\right|^{p(x)}\right)^{1 / p_{-}},\left(\int_{\mathbb{R}^{N}}\left|v_{n}\right|^{p(x)}\left|\nabla \varphi_{\varepsilon}\right|^{p(x)}\right)^{1 / p_{+}}\right\},
\end{aligned}
$$


410

C.O. Aves - M.C. Ferreira

where $p^{\prime}(x)=p(x) /(p(x)-1)$ for all $x \in \mathbb{R}^{N}$. Therefore, by Lebesgue's domenoted convergence theorem,

$$
\begin{aligned}
\left.\limsup _{n}\left|\int_{\mathbb{R}^{N}} v_{n}\right| \nabla v_{n}\right|^{p(x)-2} \nabla v_{n} \cdot \nabla \varphi_{\varepsilon} \mid \\
\leq C \max \left\{\left(\int_{\mathbb{R}^{N}}|v|^{p(x)}\left|\nabla \varphi_{\varepsilon}\right|^{p(x)}\right)^{1 / p_{-}},\left(\int_{\mathbb{R}^{N}}|v|^{p(x)}\left|\nabla \varphi_{\varepsilon}\right|^{p(x)}\right)^{1 / p_{+}}\right\} .
\end{aligned}
$$

Furthermore, by Hölder's inequality

$$
\int_{\mathbb{R}^{N}}|v|^{p(x)}\left|\nabla \varphi_{\varepsilon}\right|^{p(x)} \leq\left.\left.\left.\left. C|| v\right|^{p(x)}\right|_{L^{N /(N-p(x))}\left(B_{2 \varepsilon}\left(x_{i}\right)\right)}|| \nabla \varphi_{\varepsilon}\right|^{p(x)}\right|_{L^{N / p(x)}\left(B_{2 \varepsilon}\left(x_{i}\right)\right)} .
$$

Once that

$$
\int_{B_{2 \varepsilon}\left(x_{i}\right)}\left|\nabla \varphi_{\varepsilon}\right|^{N}=\int_{B_{2}(0)}|\nabla \varphi|^{N}
$$

we derive

$$
\begin{aligned}
& \|\left.\left.\nabla \varphi_{\varepsilon}\right|^{p(x)}\right|_{L^{N / p(x)}\left(B_{2 \varepsilon}\left(x_{i}\right)\right)} \\
& \quad \leq \max \left\{\left(\int_{B_{2 \varepsilon}\left(x_{i}\right)}\left|\nabla \varphi_{\varepsilon}\right|^{N}\right)^{1 /(N / p)-},\left(\int_{B_{2 \varepsilon}\left(x_{i}\right)}\left|\nabla \varphi_{\varepsilon}\right|^{N}\right)^{1 /(N / p)_{+}}\right\} \leq C
\end{aligned}
$$

for some positive constant $C$, which is independent of $\varepsilon$. Thereby,

$$
\int_{\mathbb{R}^{N}}|v|^{p(x)}\left|\nabla \varphi_{\varepsilon}\right|^{p(x)} \leq\left.\left. C|| v\right|^{p(x)}\right|_{L^{N /(N-p(x))}\left(B_{2 \varepsilon}\left(x_{i}\right)\right)},
$$

and so

$$
\begin{aligned}
\underset{n}{\limsup } \mid & \int_{\mathbb{R}^{N}} v_{n}\left|\nabla v_{n}\right|^{p(x)-2} \nabla v_{n} \cdot \nabla \varphi_{\varepsilon} \mid \\
& \leq C \max \left\{\left.\left.|| v\right|^{p(x)}\right|_{L^{N /(N-p(x))}\left(B_{2 \varepsilon}\left(x_{i}\right)\right)} ^{1 / p_{-}},\left.\left.|| v\right|^{p(x)}\right|_{L^{N /(N-p(x))}\left(B_{2 \varepsilon}\left(x_{i}\right)\right)} ^{1 / p_{+}}\right\} .
\end{aligned}
$$

But,

$$
\begin{aligned}
& \left.\left.|| v\right|^{p(x)}\right|_{L^{N /(N-p(x))}\left(B_{2 \varepsilon}\left(x_{i}\right)\right)} \\
& \leq \max \left\{\left(\int_{B_{2 \varepsilon}\left(x_{i}\right)}|v|^{p^{*}(x)}\right)^{1 /(N /(N-p))},\left(\int_{B_{2 \varepsilon}\left(x_{i}\right)}|v|^{p^{*}(x)}\right)^{1 /(N /(N-p))+}\right\}
\end{aligned}
$$

from where it follows that

$$
\left.\lim _{\varepsilon \rightarrow 0} \limsup _{n}\left|\int_{\mathbb{R}^{N}} v_{n}\right| \nabla v_{n}\right|^{p(x)-2} \nabla v_{n} \nabla \varphi_{\varepsilon} \mid=0
$$

implying that

$$
\lim _{\varepsilon \rightarrow 0} \limsup _{n} \int_{\mathbb{R}^{N}} v_{n}\left|\nabla v_{n}\right|^{p(x)-2} \nabla v_{n} \nabla \varphi_{\varepsilon}=0 .
$$

Now, taking the limit as $\varepsilon \rightarrow 0$ in (3.3), we get

$$
\mathfrak{m}_{i}=\mathfrak{m}\left(x_{i}\right)=\mathfrak{n}\left(x_{i}\right)=\mathfrak{n}_{i} .
$$


Once that $p_{-}^{*} / p_{+} \leq p_{+}^{*} / p_{-}$, we have that

$$
\mathfrak{n}_{i}^{p_{+} / p_{-}^{*}} \leq\left(S^{p_{+} / p_{-}^{*}}+S^{p_{-} / p_{+}^{*}}\right) \mathfrak{m}_{i}, \quad \text { if } \mathfrak{m}_{i}<1
$$

and

$$
\mathfrak{n}_{i}^{p_{-} / p_{+}^{*}} \leq\left(S^{p_{+} / p_{-}^{*}}+S^{p_{-} / p_{+}^{*}}\right) \mathfrak{m}_{i} \quad \text { if } \mathfrak{m}_{i} \geq 1
$$

Thus, from (3.4)-(3.6), if $\mathfrak{n}_{i}>0$ for some $i \in \mathfrak{I}$, there exists $\alpha>0$, which is independent of $i$, such that

$$
\mathfrak{n}_{i} \geq \alpha
$$

Recalling that

$$
\sum_{\substack{i \in \mathfrak{I} \\ \mathfrak{m}_{i}<1}} \mathfrak{n}_{i}^{p_{+} / p_{-}^{*}}+\sum_{\substack{i \in \mathfrak{I} \\ \mathfrak{m}_{i} \geq 1}} \mathfrak{n}_{i}^{p_{-} / p_{+}^{*}} \leq C \sum_{i \in \mathfrak{I}} \mathfrak{m}_{i}<\infty
$$

the inequality (3.7) gives $\widetilde{\mathfrak{I}}=\left\{i \in \mathfrak{I} ; \mathfrak{n}_{i}>0\right\}$ is a finite set. From this, one of the two possibilities below occurs:

(a) There exist $\mathfrak{n}_{i_{1}}, \ldots, \mathfrak{n}_{i_{s}}>0$ for a maximal $s \in \mathbb{N}$;

(b) $\mathfrak{n}_{i}=0$, for all $i \in \mathfrak{I}$.

We begin analyzing (a). For this, choose $0<\varepsilon_{0}<1$ sufficiently small such that

$$
B_{\varepsilon_{0}}\left(x_{1}\right), \ldots, B_{\varepsilon_{0}}\left(x_{s}\right) \subset B_{\frac{1}{\varepsilon_{0}}}(0) \quad \text { and } \quad B_{\varepsilon_{0}}\left(x_{i}\right) \cap B_{\varepsilon_{0}}\left(x_{j}\right)=\emptyset, \quad i \neq j,
$$

where $x_{1}, \ldots, x_{s}$ are the singular points related to $\mathfrak{n}_{i_{1}}, \ldots, \mathfrak{n}_{i_{s}}$, respectively. We set

$$
\psi_{\varepsilon}(x)=\varphi(\varepsilon x)-\sum_{i=1}^{s} \varphi\left(\frac{x-x_{i}}{\varepsilon}\right) \quad \text { for all } x \in \mathbb{R}^{N} .
$$

Then, for $0<\varepsilon<\varepsilon_{0} / 2$,

$$
\psi_{\varepsilon}(x)= \begin{cases}0 & \text { if } x \in \bigcup_{i=1}^{s} B_{\varepsilon / 2}\left(x_{i}\right) \\ 1 & \text { if } x \in A_{\varepsilon}=B_{1 / \varepsilon}(0) \backslash \bigcup_{i=1}^{s} B_{2 \varepsilon}\left(x_{i}\right),\end{cases}
$$

and

$$
\operatorname{supp} \psi_{\varepsilon} \subset \overline{B_{2 / \varepsilon}(0)} \backslash \bigcup_{i=1}^{s} B_{\varepsilon / 2}\left(x_{i}\right)
$$

loading to

$$
\int_{\mathbb{R}^{N}}\left|v_{n}\right|^{p^{*}(x)} \psi_{\varepsilon} \rightarrow \int_{\mathbb{R}^{N}}|v|^{p^{*}(x)} \psi_{\varepsilon}
$$

Since

$$
I^{\prime}\left(v_{n}\right)\left(v_{n} \psi_{\varepsilon}\right)=o_{n}(1) \text { and } I^{\prime}\left(v_{n}\right)\left(v \psi_{\varepsilon}\right)=o_{n}(1)
$$


repeating the same type of arguments for the case where the exponents are constant, we obtain

$$
\lim _{n} \int_{A_{\varepsilon}}\left(P_{n}+V(x) Q_{n}\right)=0,
$$

where

$$
P_{n}(x)=\left(\left|\nabla v_{n}\right|^{p(x)-2} \nabla v_{n}-|\nabla v|^{p(x)-2} \nabla v\right)\left(\nabla v_{n}-\nabla v\right)
$$

for all $x \in \mathbb{R}^{N}$ and for all $n \in \mathbb{N}$, and

$$
Q_{n}(x)=\left(\left|v_{n}\right|^{p(x)-2} v_{n}-|v|^{p(x)-2} v\right)\left(v_{n}-v\right)
$$

for all $x \in \mathbb{R}^{N}$ and for all $n \in \mathbb{N}$. Since

$$
P_{n}(x) \geq \begin{cases}\frac{2^{3-p_{+}}}{p_{+}}\left|\nabla v_{n}-\nabla v\right|^{p(x)} & \text { if } p(x) \geq 2, \\ \left(p_{-}-1\right) \frac{\left|\nabla v_{n}-\nabla v\right|^{2}}{\left(\left|\nabla v_{n}\right|+|\nabla v|\right)^{2-p(x)}} & \text { if } 1<p(x)<2,\end{cases}
$$

it follows that

$$
\int_{A_{\varepsilon}} P_{n} \geq C \int_{A_{\varepsilon} \cap\left\{x \in \mathbb{R}^{N} ; p(x) \geq 2\right\}}\left|\nabla v_{n}-\nabla v\right|^{p(x)} \geq 0 .
$$

Thus,

$$
\lim _{n} \int_{A_{\varepsilon} \cap\left\{x \in \mathbb{R}^{N} ; p(x) \geq 2\right\}}\left|\nabla v_{n}-\nabla v\right|^{p(x)}=0 .
$$

On the other hand, by Hölder's inequality

$$
\begin{array}{r}
\int_{A_{\varepsilon} \cap\left\{x \in \mathbb{R}^{N} ; 1<p(x)<2\right\}}\left|\nabla v_{n}-\nabla v\right|^{p(x)} \\
\leq C\left|\frac{\left|\nabla v_{n}-\nabla v\right|^{p(x)}}{\left(\left|\nabla v_{n}\right|+|\nabla v|\right)^{p(x) /(2-p(x)) 2}}\right|_{L^{2 / p(x)}\left(\widetilde{A_{\varepsilon}}\right)} \\
\cdot\left|\left(\left|\nabla v_{n}\right|+|\nabla v|\right)^{p(x)(2-p(x)) / 2}\right|_{L^{2 /(2-p(x))}\left(\widetilde{A_{\varepsilon}}\right)}
\end{array}
$$

where $\widetilde{A_{\varepsilon}}=A_{\varepsilon} \cap\left\{x \in \mathbb{R}^{N} ; 1<p(x)<2\right\}$. From relation (3.9), the right side of above inequality goes to zero. Hence,

$$
\lim _{n} \int_{A_{\varepsilon} \cap\left\{x \in \mathbb{R}^{N} ; 1<p(x)<2\right\}}\left|\nabla v_{n}-\nabla v\right|^{p(x)}=0 .
$$

Now (3.10) combined with (3.11) gives

$$
\lim _{n} \int_{A_{\varepsilon}}\left|\nabla v_{n}-\nabla v\right|^{p(x)}=0 .
$$

The same arguments can be used to prove that

$$
\lim _{n} \int_{A_{\varepsilon}} V(x)\left|v_{n}-v\right|^{p(x)}=0 .
$$


Therefore, $v_{n} \rightarrow v$ in $W^{1, p(x)}\left(A_{\varepsilon}\right)$. The last limit yields, up to a subsequence, $\nabla v_{n}(x) \rightarrow \nabla v(x)$ almost everywhere in $A_{\varepsilon}\left(0<\varepsilon<\varepsilon_{0} / 2\right)$. Observing that

$$
\mathbb{R}^{N} \backslash\left\{x_{1}, \ldots, x_{s}\right\}=\bigcup_{\substack{n \in \mathbb{N} \\ 1 / n<\varepsilon_{0} / 2}} A_{1 / n},
$$

we conclude by a diagonal argument, that there is a subsequence of $\left(v_{n}\right)$, still denoted by itself, such that $\nabla v_{n}(x) \rightarrow \nabla v(x)$ almost everywhere in $\mathbb{R}^{N}$.

For the case (b), we consider $\psi_{\varepsilon}(x)=\varphi(\varepsilon x)$ for all $x \in \mathbb{R}^{N}$ and $A_{\varepsilon}=B_{1 / \varepsilon}(0)$, $\varepsilon>0$. Repeating the same arguments used in the case $a$ ), we have that $v_{n} \rightarrow v$ in $W^{1, p(x)}\left(B_{1 / \varepsilon}(0)\right)$. This way, there is again a subsequence of $\left(v_{n}\right)$, still denoted by itself, such that $\nabla v_{n}(x) \rightarrow \nabla v(x)$ almost everywhere in $\mathbb{R}^{N}$.

LEMmA 3.3. Let $\left(v_{n}\right)$ be a $(\mathrm{PS})_{d}$ sequence for $I$ with $v_{n} \rightarrow v$ in $W^{1, p(x)}\left(\mathbb{R}^{N}\right)$. Then, there exists a constant $M>0$, which is independent of $\lambda$ and $\mu$, such that

$$
I(v) \geq-M\left(\lambda^{\Theta_{-}}+\lambda^{\Theta_{+}}\right) .
$$

Proof. From Lemma 3.2, $I^{\prime}(v) v=0$, or equivalently,

$$
\int_{\mathbb{R}^{N}}|\nabla v|^{p(x)}+V(x) v^{p(x)}=\lambda \int_{\mathbb{R}^{N}} h(x) v^{r(x)}+\mu \int_{\mathbb{R}^{N}} v^{q(x)}+\int_{\mathbb{R}^{N}} v^{p^{*}(x)} .
$$

From this,

$$
I(v) \geq \lambda\left(\frac{1}{p_{+}}-\frac{1}{r_{-}}\right) \int_{\mathbb{R}^{N}} h(x) v^{r(x)}+\left(\frac{1}{p_{+}}-\frac{1}{p_{-}^{*}}\right) \int_{\mathbb{R}^{N}} v^{p^{*}(x)},
$$

which together with Young's inequality implies that for all $\varepsilon>0$,

$$
\begin{aligned}
I(v) \geq & \varepsilon\left(\frac{1}{p_{+}}-\frac{1}{r_{-}}\right) \int_{\mathbb{R}^{N}} v^{p^{*}(x)} \\
& +\left(\frac{1}{p_{+}}-\frac{1}{r_{-}}\right) \int_{\mathbb{R}^{N}} C(\varepsilon, x) \lambda^{\Theta(x)} h^{\Theta(x)}+\left(\frac{1}{p_{+}}-\frac{1}{p_{-}^{*}}\right) \int_{\mathbb{R}^{N}} v^{p^{*}(x)},
\end{aligned}
$$

where

Fixing

$$
C(\varepsilon, x)=\frac{1}{\Theta(x)\left(\varepsilon \frac{p^{*}(x)}{r(x)}\right)^{r(x) \Theta(x) / p^{*}(x)}} .
$$

$$
0<\varepsilon<\min \left\{1,\left(\frac{1}{r_{-}}-\frac{1}{p_{+}}\right)^{-1}\left(\frac{1}{p_{+}}-\frac{1}{p_{-}^{*}}\right)\right\},
$$

it follows that $I(u) \geq-M\left(\lambda^{\Theta_{-}}+\lambda^{\Theta_{+}}\right)$, where

$$
M=\frac{1}{\Theta_{-} \varepsilon^{\Theta_{+}-1}}\left(\frac{1}{r_{-}}-\frac{1}{p_{+}}\right) \int_{\mathbb{R}^{N}} h^{\Theta(x)} .
$$

The next result is an important step to understand the behavior of the (PS) sequences of $I$. 
LEMmA 3.4. Let $\left(v_{n}\right)$ be a bounded sequence in $W^{1, p(x)}\left(\mathbb{R}^{N}\right)$ such that $v_{n}(x) \rightarrow$ $v(x)$ and $\nabla v_{n}(x) \rightarrow \nabla v(x)$ almost everywhere in $\mathbb{R}^{N}$. Then:

(a) $I\left(v_{n}\right)-I_{\mu}\left(v_{n}-v\right)-I(v)=o_{n}(1)$, and

(b) $I^{\prime}\left(v_{n}\right)-I_{\mu}^{\prime}\left(v_{n}-v\right)-I^{\prime}(v)=o_{n}(1)$.

Consequently, if $\left(v_{n}\right)$ is a (PS $)_{d}$ sequence for I with weak limit $v \in W^{1, p(x)}\left(\mathbb{R}^{N}\right)$, setting $w_{n}=v_{n}-v$, we have that for some subsequence, $\left(w_{n}\right)$ is a $(\mathrm{PS})_{d-I(v)}$ sequence for $I_{\mu}$.

Proof. From definitions of $I$ and $I_{\mu}$, we derive that

$$
\begin{aligned}
I\left(v_{n}\right) & -I_{\mu}\left(v_{n}-v\right)-I(v) \\
= & \int_{\mathbb{R}^{N}} \frac{1}{p(x)}\left(\left|\nabla v_{n}\right|^{p(x)}-\left|\nabla v_{n}-\nabla u\right|^{p(x)}-|\nabla v|^{p(x)}\right) \\
& +\int_{\mathbb{R}^{N}} \frac{V(x)}{p(x)}\left(v_{n}^{p(x)}-\left|v_{n}-v\right|^{p(x)}-v^{p(x)}\right) \\
& -\mu \int_{\mathbb{R}^{N}} \frac{1}{q(x)}\left(v_{n}^{q(x)}-\left|v_{n}-v\right|^{q(x)}-v^{q(x)}\right) \\
& -\int_{\mathbb{R}^{N}} \frac{1}{p^{*}(x)}\left(v_{n}^{p^{*}(x)}-\left|v_{n}-v\right|^{p^{*}(x)}-v^{p^{*}(x)}\right)-\lambda \int_{\mathbb{R}^{N}} \frac{h(x)}{r(x)}\left(v_{n}^{r(x)}-v^{r(x)}\right) .
\end{aligned}
$$

By Propositions 2.5 and 2.6, we observe that the right side of the last inequality is $o_{n}(1)$, and so,

$$
I\left(v_{n}\right)-I_{\mu}\left(v_{n}-v\right)-I(v)=o_{n}(1),
$$

showing (a).

Now, to prove (b), we fix $\varphi \in W^{1, p(x)}\left(\mathbb{R}^{N}\right)$ with $\|\varphi\|=1$. Using Hölder's inequality together with Sobolev's embedding, it follows that there is a positive constant $C$ such that

$$
\left[I^{\prime}\left(v_{n}\right)-I_{\mu}^{\prime}\left(v_{n}-v\right)-I^{\prime}(v)\right] \varphi \leq C\left(A_{1}(n)+A_{2}(n)+A_{3}(n)+A_{4}(n)+A_{5}(n)\right)
$$

where

$$
\begin{aligned}
& A_{1}(n)=\left.|| \nabla v_{n}\right|^{p(x)-2} \nabla v_{n}-\left|\nabla v_{n}-\nabla v\right|^{p(x)-2}\left(\nabla v_{n}-\nabla v\right)-\left.|\nabla v|^{p(x)-2} \nabla v\right|_{p^{\prime}(x)}, \\
& A_{2}(n)=\left|v_{n}^{p(x)-2} v_{n}-\right| v_{n}-\left.v\right|^{p(x)-2}\left(v_{n}-v\right)-\left.v^{p(x)-2} v\right|_{p^{\prime}(x)}, \\
& A_{3}(n)=\mu\left|v_{n}^{q(x)-2} v_{n}-\right| v_{n}-\left.v\right|^{q(x)-2}\left(v_{n}-v\right)-\left.v^{q(x)-2} v\right|_{q^{\prime}(x)}, \\
& A_{4}(n)=\left|v_{n}^{p^{*}(x)-2} v_{n}-\right| v_{n}-\left.v\right|^{p^{*}(x)-2}\left(v_{n}-v\right)-\left.v^{p^{*}(x)-2} v\right|_{p^{* \prime}(x)},
\end{aligned}
$$

and

$$
A_{5}(n)=\lambda \int_{\mathbb{R}^{N}} h(x)\left|\left(v_{n}^{r(x)-1}-v^{r(x)-1}\right) \varphi\right| .
$$

From Proposition 2.5, $A_{i}(n)=o_{n}(1)$ for $i=1,2,3,4$. Related to $A_{5}(n)$, we have that

$$
\int_{\mathbb{R}^{N}} h(x)\left|\left(v_{n}^{r(x)-1}-v^{r(x)-1}\right) \varphi\right|=\int_{\mathbb{R}^{N}} h^{1 / r^{\prime}(x)}\left|v_{n}^{r(x)-1}-v^{r(x)-1}\right| h^{1 / r(x)}|\varphi| .
$$


Since

$$
h^{1 / r^{\prime}(x)}\left|v_{n}^{r(x)-1}-v^{r(x)-1}\right| \in L^{r^{\prime}(x)}\left(\mathbb{R}^{N}\right) \quad \text { and } \quad h^{1 / r(x)}|\varphi| \in L^{r(x)}\left(\mathbb{R}^{N}\right),
$$

by Hölder's inequality,

$$
\int_{\mathbb{R}^{N}} h(x)\left|\left(v_{n}^{r(x)-1}-v^{r(x)-1}\right) \varphi\right| \leq C\left|h^{1 / r^{\prime}(x)}\right| v_{n}^{r(x)-1}-\left.v^{r(x)-1}\right|_{r^{\prime}(x)} .
$$

Now, our goal is to prove that $\left|h^{1 / r^{\prime}(x)}\right| v_{n}^{r(x)-1}-v^{r(x)-1} \|_{r^{\prime}(x)} \rightarrow 0$, or equivalently,

$$
\int_{\mathbb{R}^{N}} h(x)\left|v_{n}^{r(x)-1}-v^{r(x)-1}\right|^{r^{\prime}(x)} \rightarrow 0 .
$$

To this end, we define $V_{n}(x)=\left|v_{n}^{r(x)-1}-v^{r(x)-1}\right|^{r^{\prime}(x)}$ for all $n \in \mathbb{N}$. Then, $V_{n}(x) \rightarrow 0$ almost everywhere in $\mathbb{R}^{N}$ and $\left(V_{n}\right)$ is bounded in $L^{p^{*}(x) / r(x)}\left(\mathbb{R}^{N}\right)$. Therefore, by Proposition 2.6, it follows that $V_{n} \rightarrow 0$ in $L^{p^{*}(x) / r(x)}\left(\mathbb{R}^{N}\right)$. Thus,

$$
\int_{\mathbb{R}^{N}} h(x) V_{n}(x) \rightarrow 0
$$

proving (3.12). Consequently,

$$
\left\|I^{\prime}\left(v_{n}\right)-I_{\mu}^{\prime}\left(v_{n}-v\right)-I^{\prime}(v)\right\|=o_{n}(1),
$$

or yet

$$
I^{\prime}\left(v_{n}\right)-I_{\mu}^{\prime}\left(v_{n}-v\right)-I^{\prime}(v)=o_{n}(1)
$$

finishing the proof.

Lemma 3.5. Suppose $\mu \geq \mu_{0}$, where $\mu_{0}$ is given in (1.2). Then, I verifies the $(\mathrm{PS})_{d}$ condition for

$$
d<c_{\mu}-M\left(\lambda^{\Theta_{-}}+\lambda^{\Theta_{+}}\right)
$$

Proof. Let $\left(v_{n}\right)$ be a $(\mathrm{PS})_{d}$ sequence for $I$ with $d$ as above. We know that there exists $v \in W^{1, p(x)}\left(\mathbb{R}^{N}\right)$ such that $v_{n} \rightarrow v$ in $W^{1, p(x)}\left(\mathbb{R}^{N}\right)$, and $v_{n}(x) \rightarrow$ $v(x)$ almost everywhere in $\mathbb{R}^{N}$. Setting $w_{n}=v_{n}-v$, by Lemma 3.4 , we see that $\left(w_{n}\right)$ is a $(\mathrm{PS})_{d-I(v)}$ sequence for $I_{\mu}$. Thus, up to a subsequence, we can assume that

$$
\int_{\mathbb{R}^{N}}\left(\left|\nabla w_{n}\right|^{p(x)}+V(x)\left|w_{n}\right|^{p(x)}\right) \rightarrow L \geq 0 .
$$

Next, we will show that $L=0$. To this end, we recall that only one of the below possibilities hold:

(a) There is $R>0$ such that

$$
\lim _{n} \sup _{y \in \mathbb{R}^{N}} \int_{B_{R}(y)}\left|w_{n}\right|^{p(x)}=0
$$

or 
(b) For each $R>0$, there are $\eta>0$, a subsequence of $\left(w_{n}\right)$, still denoted by itself, and $\left(y_{n}\right) \subset \mathbb{R}^{N}$ ( which we can suppose in $\mathbb{Z}^{N}$ ) such that

$$
\varlimsup_{n} \int_{B_{R}\left(y_{n}\right)}\left|w_{n}\right|^{p(x)} \geq \eta .
$$

We will show that (b) does not hold. Arguing by contradiction, if (b) is true, we define

$$
\widehat{w_{n}}(x)=w_{n}\left(x+y_{n}\right), \quad x \in \mathbb{R}^{N} .
$$

Then, by a simple computation, $I_{\mu}\left(\widehat{w_{n}}\right)=I_{\mu}\left(w_{n}\right)$ and $I_{\mu}^{\prime}\left(\widehat{w_{n}}\right)=o_{n}(1)$. So, $\left(\widehat{w_{n}}\right)$ is also a $(\mathrm{PS})_{d-I(v)}$ sequence for $I_{\mu}$. Let $\widehat{w} \in W^{1, p(x)}\left(\mathbb{R}^{N}\right) \backslash\{0\}$ the weak limit of $\widehat{w_{n}}$. Since $I_{\mu}^{\prime}(\widehat{w})=0$ and $\widehat{w} \neq 0$, it follows from the definition of $c_{\mu}$ that

$$
\begin{aligned}
c_{\mu} \leq & I_{\mu}(\widehat{w})=I_{\mu}(\widehat{w})-\frac{1}{p_{+}} I_{\mu}^{\prime}(\widehat{w}) \widehat{w} \\
\leq & \frac{\lim }{n}\left(\int_{\mathbb{R}^{N}}\left(\frac{1}{p(x)}-\frac{1}{p_{+}}\right)\left(\left|\nabla \widehat{w_{n}}\right|^{p(x)}+V(x)\left|\widehat{w_{n}}\right|^{p(x)}\right)\right. \\
& \left.+\mu \int_{\mathbb{R}^{N}}\left(\frac{1}{p_{+}}-\frac{1}{q(x)}\right)\left|\widehat{w_{n}}\right|^{q(x)}+\int_{\mathbb{R}^{N}}\left(\frac{1}{p_{+}}-\frac{1}{p^{*}(x)}\right) \mid{\widehat{w_{n}}}^{p^{*}(x)}\right) \\
= & \frac{\lim }{n}\left(I_{\mu}\left(\widehat{w_{n}}\right)-\frac{1}{p_{+}} I_{\mu}^{\prime}\left(\widehat{w_{n}}\right) \widehat{w_{n}}\right)=d-I(v) \leq d+M\left(\lambda^{\Theta}+\lambda^{\Theta_{+}}\right) .
\end{aligned}
$$

Thus, $c_{\mu}-M\left(\lambda^{\Theta_{-}}+\lambda^{\Theta_{+}}\right) \leq d$, which is a contradiction with the hypothesis on $d$. Therefore, (b) does not hold. Then (a) holds, and by Lemma 3.1 in [22], $w_{n} \rightarrow 0$ in $L^{q(x)}\left(\mathbb{R}^{N}\right)$, or equivalently,

$$
\int_{\mathbb{R}^{N}}\left|w_{n}\right|^{q(x)} \rightarrow 0
$$

Since $I_{\mu}^{\prime}\left(w_{n}\right) w_{n}=o_{n}(1)$, we derive that

$$
\int_{\mathbb{R}^{N}}\left|w_{n}\right|^{p^{*}(x)} \rightarrow L .
$$

By (3.13),

$$
\begin{aligned}
d-I(v)+o_{n}(1) & =I_{\mu}\left(w_{n}\right)+\mu \int_{\mathbb{R}^{N}} \frac{1}{q(x)}\left|w_{n}\right|^{q(x)} \\
& =\int_{\mathbb{R}^{N}} \frac{1}{p(x)}\left(\left|\nabla w_{n}\right|^{p(x)}+V(x)\left|w_{n}\right|^{p(x)}\right)-\int_{\mathbb{R}^{N}} \frac{1}{p^{*}(x)}\left|w_{n}\right|^{p^{*}(x)}
\end{aligned}
$$

and so,

$$
d-I(v)+o_{n}(1) \geq \frac{1}{p_{+}} \int_{\mathbb{R}^{N}}\left(\left|\nabla w_{n}\right|^{p(x)}+V(x)\left|w_{n}\right|^{p(x)}\right)-\frac{1}{p_{-}^{*}} \int_{\mathbb{R}^{N}}\left|w_{n}\right|^{p^{*}(x)} .
$$

Taking the limit of $n \rightarrow+\infty$ in the last inequality, we see that

$$
d-I(v) \geq \frac{1}{p_{+}} L-\frac{1}{p_{-}^{*}} L=\gamma L .
$$


In this moment, it is very important to recall that $I(v) \geq-M\left(\lambda^{\Theta_{-}}+\lambda^{\Theta_{+}}\right)$. Then, by the hypothesis on $d$,

$$
d-I(v)<c_{\mu} .
$$

On the other hand, since $\mu \geq \mu_{0}$, the last inequality combined with (1.2) leads to $d-I(v)<\nu / 2 K^{p_{+}}$. Using this information, we get $\left\|w_{n}\right\| \leq 1 / K<1$ for all $n \geq n_{0}$ and so, $\left|w_{n}\right|_{p^{*}(x)} \leq 1$ for all $n \geq n_{0}$. The above inequalities show that

$$
\left(\int_{\mathbb{R}^{N}}\left|w_{n}\right|^{p^{*}(x)}\right)^{1 / p_{-}^{*}} \leq K\left(\int_{\mathbb{R}^{N}}\left|\nabla w_{n}\right|^{p(x)}+V(x)\left|v_{n}\right|^{p(x)}\right)^{1 / p_{+}},
$$

for all $n \geq n_{0}$. Taking the limit of $n \rightarrow+\infty$, we derive $L^{1 / p_{-}^{*}} \leq K L^{1 / p_{+}}$. Supposing by contradiction that $L>0$, we obtain

$$
L \geq\left(\frac{1}{K}\right)^{1 / \gamma} .
$$

Combining (3.14) with (3.16), it follows that $d-I(v) \geq \gamma L \geq \gamma(1 / K)^{1 / \gamma}$, which is a contradiction, once that (3.15) and (1.2) imply that $d-I(v)<\gamma(1 / K)^{1 / \gamma}$. Thereby, $L=0$.

\section{Existence of solution with positive energy}

In this section, we will show the existence of a solution via Mountain Pass Theorem. Our first lemma establishes that $I$ verifies the mountain pass geometry.

Lemma 4.1. For each $\mu>0$, there exists $\lambda_{1}=\lambda_{1}(\mu)>0$ such that I satisfies the mountain pass geometry, if $\lambda \in\left(0, \lambda_{1}\right)$.

Proof. First of all, we observe that, for all $u \in W^{1, p(x)}\left(\mathbb{R}^{N}\right)$,

$$
\begin{aligned}
I(u) \geq \frac{1}{p_{+}} \int_{\mathbb{R}^{N}}\left(|\nabla u|^{p(x)}+V(x)|u|^{p(x)}\right) & -\frac{\lambda}{r_{-}} \int_{\mathbb{R}^{N}} h(x)|u|^{r(x)} \\
& -\frac{\mu}{q_{-}} \int_{\mathbb{R}^{N}}|u|^{q(x)}-\frac{1}{p_{-}^{*}} \int_{\mathbb{R}^{N}}|u|^{p^{*}(x)} .
\end{aligned}
$$

By Sobolev's embedding, there are $C_{1}, C_{2}>0$ such that

$$
|u|_{q(x)} \leq C_{1}\|u\| \quad \text { and } \quad|u|_{p^{*}(x)} \leq C_{2}\|u\|, \quad \text { for all } u \in W^{1, p(x)}\left(\mathbb{R}^{N}\right) .
$$

If we suppose that $\|u\|<m=\min \left\{1,1 / C_{1}, 1 / C_{2}\right\}$, then

$$
\|u\|<1,|u|_{q(x)}<1 \quad \text { and } \quad|u|_{p^{*}(x)}<1 .
$$

The above inequalities yield

$$
I(u) \geq \frac{1}{p_{+}}\|u\|^{p_{+}}-\lambda C_{3}|h|_{\Theta(x)}\|u\|^{r_{-}-}-\mu C_{4}\|u\|^{q_{-}}-C_{5}\|u\|^{p_{-}^{*}}, \quad \text { if }\|u\|<m .
$$

Since $p_{+}<q_{-}, p_{-}^{*}$, we can choose $R=R(\mu) \in(0, m)$ such that

$$
\frac{1}{p_{+}} R^{p_{+}}-\mu C_{4} R^{q_{-}}-C_{5} R^{p_{-}^{*}} \geq \frac{1}{2 p_{+}} R^{p_{+}} .
$$


So, if $\|u\|=R$,

$$
I(u) \geq \frac{1}{2 p_{+}} R^{p_{+}}-\lambda C_{3}|h|_{\Theta(x)} R^{r_{-}} .
$$

Now, we choose $\lambda_{1}=\lambda_{1}(\mu)>0$ such that

$$
\frac{1}{2 p_{+}} R^{p_{+}}-\lambda_{1} C_{3}|h|_{\Theta(x)} R^{r_{-}}=\beta>0 .
$$

Consequently, if $\lambda \in\left(0, \lambda_{1}\right)$, we have that $I(u) \geq \beta$, for $\|u\|=R$, showing that the first geometry is satisfied. For the second geometry, we fix $u \in W^{1, p(x)}\left(\mathbb{R}^{N}\right)$ with $u^{+} \neq 0$. Then, for $t>1$,

$$
\begin{aligned}
I(t u) \leq t^{p_{+}} \int_{\mathbb{R}^{N}} \frac{1}{p(x)}( & \left.|\nabla u|^{p(x)}+V(x)|u|^{p(x)}\right)-t^{r_{-}} \int_{\mathbb{R}^{N}} \frac{h(x)}{r(x)}\left(u^{+}\right)^{r(x)} \\
& -t^{q_{-}} \int_{\mathbb{R}^{N}} \frac{1}{q(x)}\left(u^{+}\right)^{q(x)}-t^{p_{-}^{*}} \int_{\mathbb{R}^{N}} \frac{1}{p^{*}(x)}\left(u^{+}\right)^{p^{*}(x)},
\end{aligned}
$$

from where it follows that $\lim _{t \rightarrow \infty} I(t u)=-\infty$. From this, we observe that the second geometry follows choosing $e=t_{0} u$ with $t_{0}>R /\|u\|$ and $I\left(t_{0} u\right) \leq 0$.

Lemma 4.2. For each $\mu \geq \mu_{0}$, there exists $0<\lambda_{2}=\lambda_{2}(\mu) \leq \lambda_{1}$, with $\lambda_{1}$ given in Lemma 4.1, such that the mountain pass level $c$ of I satisfies

$$
c<c_{\mu}-M\left(\lambda^{\Theta_{-}}+\lambda^{\Theta_{+}}\right), \quad \text { for all } \lambda \in\left(0, \lambda_{2}\right) .
$$

Proof. For each $\mu \geq \mu_{0}$, we know that there is $\Psi \in W^{1, p(x)}\left(\mathbb{R}^{N}\right) \backslash\{0\}$ with $\Psi \geq 0$ such that $I_{\mu}(\Psi)=c_{\mu}$ and $I_{\mu}^{\prime}(\Psi)=0$. In what follows, fix $\delta_{1}>0$ such that

$$
c_{\mu}-M\left(\lambda^{\Theta_{-}}+\lambda^{\Theta_{+}}\right)>\frac{c_{\mu}}{2}, \quad \text { for all } \lambda \in\left(0, \delta_{1}\right) .
$$

Since for $t>0$ sufficiently small

$$
I(t \Psi) \leq t^{p_{-}} \int_{\mathbb{R}^{N}} \frac{1}{p(x)}\left(|\nabla u|^{p(x)}+V(x)|u|^{p(x)}\right),
$$

there is $t_{0}>0$, which is independent of $\lambda$, such that $I(t \Psi) \leq c_{\mu} / 2$, for all $t \in\left[0, t_{0}\right]$. Therefore, for each $\lambda \in\left(0, \delta_{1}\right)$,

$$
I(t \Psi) \leq \frac{c_{\mu}}{2}<c_{\mu}-M\left(\lambda^{\Theta_{-}}+\lambda^{\Theta_{+}}\right), \quad \text { for all } t \in\left[0, t_{0}\right] .
$$

On the other hand, using the fact that $\Psi \geq 0$, we have that

$$
I(t \Psi)=I_{\mu}(t \Psi)-\lambda \int_{\mathbb{R}^{N}} \frac{h(x)}{r(x)}(t \Psi)^{r(x)} \quad \text { for } t \geq 0,
$$

from where it follows that

$$
I(t \Psi) \leq c_{\mu}-\lambda \min \left\{t^{r_{-}}, t^{r_{+}}\right\} \int_{\mathbb{R}^{N}} \frac{h(x)}{r(x)} \Psi^{r(x)} .
$$

In particular, for $t \geq t_{0}$,

$$
I(t \Psi) \leq c_{\mu}-\lambda \min \left\{t_{0}^{r_{-}}, t_{0}^{r_{+}}\right\} \int_{\mathbb{R}^{N}} \frac{h(x)}{r(x)} \Psi^{r(x)} .
$$


Fixing $\delta_{2}>0$ such that

$$
\lambda^{\Theta_{-}-1}+\lambda^{\Theta_{+}-1}<\frac{\min \left\{t_{0}^{r_{-}}, t_{0}^{r_{+}}\right\}}{M} \int_{\mathbb{R}^{N}} \frac{h(x)}{r(x)} \Psi^{r(x)}, \quad \text { for all } \lambda \in\left(0, \delta_{2}\right),
$$

we have that $\sup _{t \geq t_{0}} I(t \Psi)<c_{\mu}-M\left(\lambda^{\Theta_{-}}+\lambda^{\Theta_{+}}\right)$, if $\lambda \in\left(0, \delta_{2}\right)$. Setting $\lambda_{2}=$ $\min \left\{\lambda_{1}, \delta_{1}, \delta_{2}\right\}$, we obtain by the previous estimates,

$$
\sup _{t \geq 0} I(t \Psi)<c_{\mu}-M\left(\lambda^{\Theta_{-}}+\lambda^{\Theta_{+}}\right) \quad \text { for all } \lambda \in\left(0, \lambda_{2}\right) .
$$

Once that $c \leq \sup _{t \geq 0} I(t \Psi)$, for $\lambda \in\left(0, \lambda_{2}\right)$, it follows that $c<c_{\mu}-M\left(\lambda^{\Theta_{-}}+\lambda^{\Theta_{+}}\right)$, finishing the proof of the lemma.

TheOREm 4.3. For each $\mu \geq \mu_{0}$, there exists $\lambda^{\star}=\lambda^{\star}(\mu)>0$ such that problem $(\mathrm{P})$ has a solution with positive energy, for all $\lambda \in\left(0, \lambda^{\star}\right)$.

Proof. Since $\mu \geq \mu_{0}$, by Lemma 3.5, the functional $I$ verifies the $(\mathrm{PS})_{d}$ condition for $d<c_{\mu}-M\left(\lambda^{\Theta_{-}}+\lambda^{\Theta_{+}}\right)$.

In what follows, we fix $\lambda^{\star}=\lambda_{2}$, where $\lambda_{2}$ was obtained in Lemma 4.2. From this, if $\lambda \in\left(0, \lambda^{\star}\right)$, by Lemma $4.1, I$ has the mountain pass geometry, and by Lemma 4.2, the mountain pass level $c$ satisfies $0<c<c_{\mu}-M\left(\lambda^{\Theta_{-}}+\lambda^{\Theta_{+}}\right)$. Thereby, $I$ satisfies the $(\mathrm{PS})_{c}$ condition, and so, there exists $\Psi_{1} \in W^{1, p(x)}\left(\mathbb{R}^{N}\right)$ such that $I^{\prime}\left(\Psi_{1}\right)=0$ and $I\left(\Psi_{1}\right)=c>0$ showing that $\Psi_{1}$ is a nontrivial solution for $(\mathrm{P})$ with positive energy.

\section{Existence of solution with negative energy}

In this section we will show the existence of a solution with negative energy by using Ekeland's Variational Principle.

Lemma 5.1. I is bounded below in $\bar{B}_{R}(0)$, where $R>0$ is given by Lemma 4.1. Moreover,

$$
J=\inf _{u \in \bar{B}_{R}(0)} I(u)<0 .
$$

Proof. If $u \in \bar{B}_{R}(0)$, then $\|u\|<1$. Arguing like in the proof of Lemma 4.1, we obtain

$$
\begin{aligned}
|I(u)| \leq & \frac{1}{p_{-}} \int_{\mathbb{R}^{N}}\left(|\nabla u|^{p(x)}+V(x)|u|^{p(x)}\right)+\frac{\lambda}{r_{-}} \int_{\mathbb{R}^{N}} h(x)|u|^{r(x)} \\
& +\frac{\mu}{q_{-}} \int_{\mathbb{R}^{N}}|u|^{q(x)}+\frac{1}{p_{-}^{*}} \int_{\mathbb{R}^{N}}|u|^{p^{*}(x)} \\
\leq & \frac{1}{p_{-}}\|u\|^{p_{-}}+\lambda C_{3}|h|_{\Theta(x)}\|u\|^{r_{-}}+\mu C_{4}\|u\|^{q_{-}}+C_{5}\|u\|^{p_{-}^{*}} \\
\leq & \frac{1}{p_{-}} R^{p_{-}}+\lambda C_{3}|h|_{\Theta(x)} R^{r_{-}}+\mu C_{4} R^{q_{-}}+C_{5} R^{p_{-}^{*}} .
\end{aligned}
$$

From this, $I$ is bounded from below in $\bar{B}_{R}(0)$. 
Let $u \in W^{1, p(x)}\left(\mathbb{R}^{N}\right) \backslash\{0\}$ with $u^{+} \neq 0$ and $0<t<1$. Then,

$$
\begin{aligned}
I(t u) \leq t^{p_{-}} \rho(u)-\lambda t^{r+} & \int_{\mathbb{R}^{N}} \frac{h(x)}{r(x)}\left(u^{+}\right)^{r(x)} \\
& -\mu t^{q_{+}} \int_{\mathbb{R}^{N}} \frac{1}{q(x)}\left(u^{+}\right)^{q(x)}-t^{p_{+}^{*}} \int_{\mathbb{R}^{N}} \frac{1}{p^{*}(x)}\left(u^{+}\right)^{p^{*}(x)} .
\end{aligned}
$$

Since $r_{+}<p_{-}, q_{+}, p_{+}^{*}, I(t u)<0$, for $t \approx 0^{+}$, leading to $J=\inf _{u \in \bar{B}_{R}(0)} I(u)<0$.

The next result establishes the existence of a $(\mathrm{PS})_{J}$ sequence for $I$. The main tool used is Ekeland's Variational Principle and the arguments are very similar to those found in [3], this way, its proof will be omitted.

Lemma 5.2. For each $\lambda \in\left(0, \lambda_{1}\right)$, where $\lambda_{1}$ is given by Lemma 4.1 , there is $a(\mathrm{PS})_{J}$ sequence for $I$, that is, there is $\left(u_{n}\right) \subset W^{1, p(x)}\left(\mathbb{R}^{N}\right)$ satisfying

$$
I\left(u_{n}\right) \rightarrow J \quad \text { and } \quad I^{\prime}\left(u_{n}\right) \rightarrow 0
$$

Now, we are able to prove the existence of a solution with negative energy.

TheOREM 5.3. For each $\mu \geq \mu_{0}$, there exists $\lambda^{\star \star}>0$ such that problem $(\mathrm{P})$ has a solution with negative energy for all $\lambda \in\left(0, \lambda^{\star \star}\right)$.

Proof. In fact, once that $\mu \geq \mu_{0}$, by Lemma 3.5 functional $I$ verifies the (PS $)_{d}$ condition for $d<c_{\mu}-M\left(\lambda^{\Theta_{-}}+\lambda^{\Theta_{+}}\right)$. In what follows, we choose $\lambda_{3}>0$ such that $0<c_{\mu}-M\left(\lambda^{\Theta}+\lambda^{\Theta_{+}}\right)$, for all $\lambda \in\left(0, \lambda_{3}\right)$ and $\lambda^{\star \star}=\min \left\{\lambda_{1}, \lambda_{3}\right\}$. For each $\lambda \in\left(0, \lambda^{\star \star}\right)$, it follows from Lemma 5.2 that there exists a $(\mathrm{PS})_{J}$ sequence $\left(u_{n}\right)$ for $I$, where $J=\inf _{u \in \bar{B}_{R}(0)} I(u)$. By Lemma 5.1, we have $J<0$, then $I$ verifies the $(\mathrm{PS})_{J}$ condition. From this, there exists $\Psi_{2} \in W^{1, p(x)}\left(\mathbb{R}^{N}\right)$ such that $I^{\prime}\left(\Psi_{2}\right)=0$ and $I\left(\Psi_{2}\right)=J<0$. Hence, $\Psi_{2}$ is a nontrivial solution for $(\mathrm{P})$ with negative energy.

\section{Final comments}

Regarding to the problem

$(\mathrm{P})_{*} \quad\left\{\begin{array}{l}-\Delta_{p(x)} u+V(x)|u|^{p(x)-2} u \\ \quad=\lambda h(x)|u|^{r(x)-2} u+\mu|u|^{q(x)-2} u+|u|^{p^{*}(x)-2} u, \quad \mathbb{R}^{N}, \\ u \in W^{1, p(x)}\left(\mathbb{R}^{N}\right),\end{array}\right.$

repeating the same arguments used by Azorero and Alonso [29], we can prove that there exists $\mu^{*}>0$ with the following property: for each $\mu \geq \mu^{*}$, there is $\lambda_{\mu}>0$ such that $(\mathrm{P})_{*}$ has infinitely many solutions with negative energy, if $\lambda \in\left(0, \lambda_{\mu}\right)$. This result is obtained using the concept and properties of genus and working with a truncation of the energy functional corresponding to $(\mathrm{P})_{*}$. 


\section{REFERENCES}

[1] E. Acerbi and G. Mingione, Regularity results for stationary electrorheological fluids, Arch. Rational Mech. Anal. 164 (2002), 213-259.

[2] _ Regularity results for electrorheological fluids: stationary case, C.R. Math. Acad. Sci. Paris334 (2002), 817-822.

[3] C.O. ALves, Multiple positive solutions for equations involving critical Sobolev exponent in $\mathbb{R}^{N}$, Electron. J. Differential Equations 1997 (1997), 1-10.

[4] _ Existence of positive solutions for a problem with lack of compactness involving the p-Laplacian, Nonlinear Anal. 51 (2002), 1187-1206.

[5] _ Existence of solutions for a degenerate $p(x)$-Laplacian equation in $\mathbb{R}^{N}$, J. Math. Anal. Appl. 345 (2008), 731-742.

[6] _ Existence of radial solutions for a class of $p(x)$-Laplacian equations with critical growth, Differential Integral Equations 23 (2010), 113-123.

[7] C.O. Alves AND M.C. FerReira, Nonlinear perturbations of a $p(x)$-Laplacian equation with critical growth in $\mathbb{R}^{N}$, Math. Nachr. 287 (2014), 849-868.

[8] C.O. Alves And M.A.S. Souto, Existence of solutions for a class of problems in $\mathbb{R}^{N}$ involving $p(x)$-Laplacian, Progr. Nonlinear Differential Equations Appl. 66 (2005), 1732.

[9] A. Ambrosetti, H. Brézis and G. Cerami, Combined effects of concave and convex nonlinearities in some elliptic problems, J. Funct. Anal. 122 (1994), 519-543.

[10] S.N. Antontsev And J.F. Rodrigues, On stationary thermo-rheological viscous flows, Ann. Univ. Ferrara, Sez. VII Sc. Mat. 52 (2006), 19-36.

[11] T. Bartsch And M. Willem, On an elliptic equation with concave and convex nonlinearities, Proc. Amer. Math. Soc. 123 (1995), 3555-3561.

[12] A. Cabada And R. Pouso, Existence theory for functional p-Laplacian equations with variable exponents, Nonlinear Anal. 52 (2003), 557-572.

[13] D.M. CAO, G.B Li AND H.S. ZhOU, Multiple solutions for non-homogeneous elliptic equations with critical Sobolev exponents, Proc. Roy. Soc. Edinburgh Sect A 124 (1994), $1177-1191$.

[14] A. Chambolle And P.L. Lions, Image recovery via total variation minimization and related problems, Numer. Math. 76 (1997), 167-188.

[15] Y. Chen, S. Levine And M. RaO, Variable exponent, linear growth functionals in image restoration, SIAM J. Appl. Math. 66 (2006), 1383-1406.

[16] X.L. FAN, On the sub-supersolution method for $p(x)$-Laplacian equations, J. Math. Anal. Appl. 330 (2007), 665-682.

$[17] \_, p(x)$-Laplacian equations in $\mathbb{R}^{N}$ with periodic data and nonperiodic perturbations, J. Math. Anal. Appl. 341 (2008), 103-119.

[18] X.L FAN AND X. HAN, Existence and multiplicity of solutions for $p(x)$-Laplacian equations in $\mathbb{R}^{N}$, Nonlinear Anal. 59 (2004), 173-188.

[19] X.L. Fan, J.S. Shen And D. ZhaO, Sobolev embedding theorems for spaces $W^{k, p(x)}(\Omega)$, J. Math. Anal. Appl. 262 (2001), 749-760.

[20] X.L. FAn AND Q.H. Zhang, Existence of solutions for $p(x)$-Laplacian Dirichlet problem, Nonlinear Anal. 52 (2003), 1843-1852.

[21] X.L. Fan and D. ZhaO, On the Spaces $L^{p(x)}(\Omega)$ and $W^{1, p(x)}(\Omega)$, J. Math. Anal. Appl. 263 (2001), 424-446.

[22] X.L. FAn, Y. ZhaO AND D. ZhaO, Compact imbedding theorems with symmetry of StraussLions type for the space $W^{1, p(x)}\left(\mathbb{R}^{N}\right)$, J. Math. Anal. Appl. 255 (2001), 333-348. 
[23] _ Nodal solutions of $p(x)$-Laplacian equations, Nonlinear Anal. 67 (2007), 28592868.

[24] J. Fernández Bonder, N. Saintier And A. Silva, On the Sobolev embedding theorem for variable exponent spaces in the critical range, J. Differential Equations 253 (2012), 1604-1620.

[25] Existence of solution to a critical equation with variable exponent, Ann. Acad. Sci. Fenn. Math 37 (2012), 579-594.

[26] J. FERnández Bonder And A. Silva, The concentration-compactness principle for variable exponent spaces and applications, Electron. J. Differential Equations 2010 (2010), $1-18$.

[27] Y. FU AND X. ZHANG, Multiple solutions for a class of $p(x)$-Laplacian equations in involving the critical exponent, Proc. Roy. Soc. Edinburgh Sect. A 466 (2010), 1667-1686.

[28] SOLUTIONS OF $p(x)$-LAPLACIAN EQUATIONS WITH CRITICAL EXPONENT AND PERTUbations in $\mathbb{R}^{N}$, Electron. J. Differential Equations 2012 (2012), 1-14.

[29] J. Garcia Azorero and I.Peral Alonzo, Multiplicity of solutions for elliptic problems with critical exponent or with nonsymmetric term, Trans. Amer. Math. Soc. 323 (2) (1991), 887-895.

[30] J.V. GonÇAlves And C.O. Alves, Existence of positive solutions for m-Laplacian equations in $\mathbb{R}^{N}$ involving critical exponents, Nonlinear Anal. 32 (1998), 53-70.

[31] M. Gueda And L. Veron, Quasilinear elliptic equations involving critical Sobolev exponents, Nonlinear Anal. 13 (1989), 879-902.

[32] O. KAVIAN, Introduction à la théorie de points critiques et applications aux problèmes elliptiques, Springer-Verlag France, Paris, 1993.

[33] C. Mercuri And M. Willem, A global compactness result for the p-Laplacian involving critical nonlinearities, Discrete Contin. Dyn. Syst. 28 (2010), 469-493.

[34] M. MihĂILESCU AND V. RĂDUlEsCU, On a nonhomogeneous quasilinear eigenvalue problem in Sobolev spaces with variable exponent, Proc. Amer. Math. Soc. 135 (9) (2007), 2929-2937, (electronic).

[35] J. Musielak, Orlicz Spaces and Modular Spaces, Lecture Notes in Math., vol. 1034, Springer-Verlag, Berlin, 1983.

[36] X. PAN, Positive solutions of the elliptic equation $\Delta u+u^{(n+2) /(n-2)}+K(x) u^{q}=0$ in $\mathbb{R}^{N}$, J. Math. Anal. Appl. 172 (1993), 323-338.

[37] M. RuzicKA, Electrorheological Fluids: Modeling and Mathematical Theory, Lecture Notes in Mathematics, vol. 1748, Springer-Verlag, Berlin, 2000.

[38] X. SHANG AND Z. WANG, Existence of solutions to discontinuos $p(x)$-Laplacian problems with critical exponents, Electron. J. Differential Equations 2012 (2012), 1-12.

[39] G. Tarantello, On nonhomogenous elliptic equations involving critical Sobolev exponent, Ann. Inst. H. Poincaré Anal. Non. Lineáire 9 (1992), 243-261.

[40] M. Willem, Minimax Theorems, Birkhäuser, Boston, MA, 1996.

Claudianor O. Alves and Marcelo C. Ferreira

Universidade Federal de Campina Grande

Unidade Acadêmica de Matemática

CEP:58429-900, Campina Grande - PB, BRAZIL

E-mail address: coalves@dme.ufcg.edu.br, marcelo@dme.ufcg.edu.br

TMNA : Volume $45-2015-\mathrm{N}^{\mathrm{O}} 2$ 